Short Communication

\title{
FUNCTIONAL ELECTRICAL STIMULATION FOR NEURO REHABILITATION. A NEW DESIGN PARADIGM
}

\section{Subramanya $\mathrm{K}^{1}{ }^{1}$, Ajithanjaya Kumar Mijar Kanakabettu ${ }^{2}$}

${ }^{1 \otimes 2}$ Department of Electrical \& Electronics Engineering,

St. Joseph Engineering College, Vamanjoor, Mangalore - 575 028, India

Correspondence

Subramanya K.

Medical Electronics Lab., Department of Electrical \& Electronics Engineering, St. Joseph Engineering College Vamanjoor, Mangalore - 575 028, India.

Tel. : +91 9986970096; Fax: +91 8242263751 E-mail : askbhat@ieee.org

\begin{abstract}
:
One of the most exciting recent advances in the neuroprosthetics field has been the application of biosignals in the design of functional electrical stimulation (FES) devices. An Electromyogram (EMG) measures the electrical activity in muscles and is often considered as ideal candidate biosignal for designing closed-loop controlled FES system. In this brief communication, we propose a novel design paradigm of a synergistic benefit of incorporating two different design principles in development of an EM G controlled FES system that hold promise for the future of rehabilitation of stroke and other neurological disorders. The proposed system will detect the residual EM G signals from the muscle and suitably adjust the stimulation current amplitude and stimulate the paralyzed muscles with a 'natural' EM G pattern envelope. We offer this design as a fruitful area for future research and clinical application.
\end{abstract}

Since 1961, functional electrical stimulation (FES) technique is used in the rehabilitation of stroke, cerebral palsy and other neurological disorders [1-3]. FES refers to clinical application of small electric impulses to stimulate peripheral nerves innervating paralyzed or weak muscles to improve the impaired motor function. FES devices are often perceived as a neurotechnological solution for restoring function in paralysis, and thus have captured the interest of engineers and clinicians alike. Both surface and implantable FES devices have been introduced to manage an important post-stroke disability, i.e., foot drop. FES helps correct foot drop and improve gait by transcutaneous stimulation of the common peroneal nerve during the swing phase of the affected leg thereby causing dorsiflexion of the foot that clears off toes and the forefoot from the ground while walking. Patients with post-stroke

\begin{tabular}{|c|}
\hline Access this article online \\
\hline Quick Response Code \\
\hline
\end{tabular}

foot drop on FES therapy experience two kinds of therapeutic effects: orthotic effect and carryover effect. The orthotic effect refers to the immediate effect on gait while the stimulation device is used. The carry over effect refers to continuation of the improvement in motor strength and motor control even after the cessation of treatment by FES. The exact mechanism of carry over effect remains an enigma. We have recently proposed a "learned paralysis" hypothesis that can account for motor relearning and the carry-over phenomenon [2].

Although a number of sophisticated FES design approaches have been introduced in past 50 years, none of them have actually proven to be clearly beneficial in clinical trials [1]. The only way to continuously improve the FES system design is to continuously come up with new and better ideas than can be shown to produce better effectiveness in clinical studies. In this brief communication, we propose a novel design paradigm of a synergistic benefit of incorporating two different design principles in the development of a neuroprosthetic that hold promise for the future of electric stimulation therapy. Synergy, a fundamental phenomenon in the natural world, can be defined as collaborative or co-operative effects produced by two or more elements or parts that are not independently obtainable. We believe that this ubiquitous phenomenon holds true even in therapeutic device 
designs, and combinations that are likely to have a synergistic effect should be an area of active research.

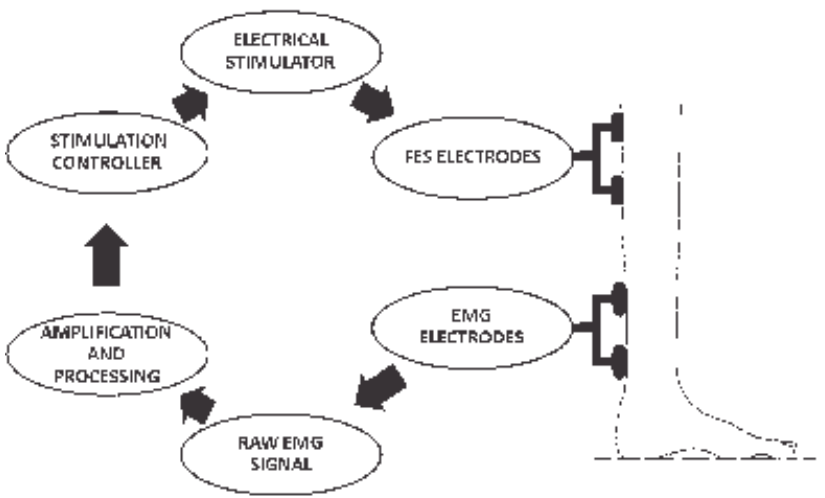

Figure 1: Block diagram of EM G controlled FES system.

The basic design of surface FES system consists of three primary components: stimulation controller unit, electrical stimulator unit and electrodes. The electrical stimulator unit receives control signals from stimulation controller unit, generates train of electrical impulses and delivers those to the nerve/muscle via electrodes, using an appropriate stimulation envelope (pattern of variation of intensity of stimulation). Conventional FES devices consist of pocket-sized stimulator unit, foot switch and two surface electrodes connected with wires. Recent studies have shown that FES systems with stimulation controller unit based on electromyography (EM G) feedback and electrical stimulator unit based on simple envelopes (trapezoidal/rectangular) are superior to conventional FES devices. In addition, FES systems with stimulation controller unit based on a simple feedback (foot switch) and electrical stimulator unit based on EMG-activation pattern envelopes have been also shown to be better than conventional ones [1]. An integration to tap potential benefits of both these designs, i.e. an "intelligent" EM Gcontrolled FES system using a EMG-based stimulation envelope, has not yet being designed. We believe that this combination system will have a synergistic action and will serve ideally to restore ambulation functions of hemiplegics. The schematic diagram of the overall system is shown in figure 1. The proposed system will detect the residual EMG signals from the muscle in real-time and suitably adjust the stimulation current amplitude and stimulate the paralyzed muscles with a 'natural' EMG pattern envelope. This system will have advantages of both EMG-controller based FES system and EMG-activation envelope based FES system, and is therefore likely to have a greater therapeutic benefit for restoring motor function and activity. We believe that the proposed bio-signal controlled intelligent FES systems holds great promises for the future of electric stimulation therapy. If this brief correspondence stimulates researchers to engage in further development of the proposed system design, it will amply serve its purpose.

\section{Acknowledgements:}

We are grateful to Prof. Manjunatha Mahadevappa of Indian Institute of Technology Kharagpur for his generous encouragement and support. We gratefully acknowledge Dr. Bikas K. Arya of Indian Institute of Technology Kharagpur for valuable advice and help. We would like to thank faculty at the department of Electrical and Electronics Engineering, St. Joseph Engineering College, Mangalore for support and guidance. Lastly, we wish to thank those who reviewed the draft manuscript for their thoughtful and helpful comments.

\section{References:}

1. Subramanya K, Pinto APJ, Kanakabettu AKM , Arya BK, M ahadevappa $M$. Surface electrical stimulation technology for stroke rehabilitation: A review of 50 years of research. J. Med. Imaging Health Inf. 2012; 2(1):1-14

2. Subramanya K, Kanakabettu AKM, Mahadevappa M. Functional electrical stimulation for stoke rehabilitation. M ed. Hypotheses 2012; 78(5):687.

3. Arya BK, Subramanya K, Mahadevappa M, Kumar R. Electrical stimulation devices for cerebral palsy: design considerations, therapeutic effects and future directions. In: Yue W, Chattopadhyay S, Lim T-C, Acharya RU (eds). Advances in Therapeutic Engineering. CRC Press, Taylor \& Francis Group, UK, 2012.

4. Arya BK, Subramanya K, M ohapatra J. Kumar R, et al. Surface EM G Analysis and Changes in Gait following Electrical Stimulation of Quadriceps Femoris and Tibialis Anterior in Children with Spastic Cerebral Palsy. Accepted for lecture at 34th Annual International IEEE EM BSConference, San Diego, USA, 2012. 